\title{
A!
}

This is an electronic reprint of the original article.

This reprint may differ from the original in pagination and typographic detail.

Tran, Nguyen; Ambos, Henrik; Jung, Alexander

\section{A Network Compatibility Condition for Compressed Sensing over Complex Networks}

\section{Published in:}

2018 IEEE Statistical Signal Processing Workshop, SSP 2018

DOI:

10.1109/SSP.2018.8450811

Published: 29/08/2018

Document Version

Peer reviewed version

Please cite the original version:

Tran, N., Ambos, H., \& Jung, A. (2018). A Network Compatibility Condition for Compressed Sensing over Complex Networks. In 2018 IEEE Statistical Signal Processing Workshop, SSP 2018 (pp. 50-54). [8450811] IEEE. https://doi.org/10.1109/SSP.2018.8450811

This material is protected by copyright and other intellectual property rights, and duplication or sale of all or part of any of the repository collections is not permitted, except that material may be duplicated by you for your research use or educational purposes in electronic or print form. You must obtain permission for any other use. Electronic or print copies may not be offered, whether for sale or otherwise to anyone who is not an authorised user. 


\title{
A NETWORK COMPATIBILITY CONDITION FOR COMPRESSED SENSING OVER COMPLEX NETWORKS
}

\author{
Nguyen Tran, Henrik Ambos, Alexander Jung
}

Department of Computer Science, Aalto University

\begin{abstract}
This paper continues our recently initiated line of work on analyzing the network Lasso (nLasso), which has been proposed as an efficient learning algorithm for massive networkstructured data sets (big data over networks). The nLasso extends the well-known Lasso estimator to network-structured datasets. In this paper we consider the nLasso using squared error loss and provide sufficient conditions on the network structure and available label information such that nLasso accurately recovers a clustered (piece-wise constant) graph signal (representing label information) from the information provided by the labels of a few data points.
\end{abstract}

Index Terms - big data over networks, compressed sensing, complex networks, network compatibility condition, network Lasso

\section{INTRODUCTION}

The datasets arising in many important applications, ranging from image processing to social network analysis, carry an intrinsic network structure which we refer to as the data graph. The nodes of the data graph represent individual data points (e.g. users in a social network), which typically carry label information called graph signal [1]. These nodes are connected by edges according to some notion of similarity. The network structure might be induced naturally by the application at hand (e.g., friendship in a social network) or obtained by learning a probabilistic graphical model for the dataset when interpreted as the realization of a random process [2-5].

On top of then network structure of, the datasets often carry additional information (e.g., labels) which we will represent by a graph signal. Since the acquisition of graph signal values (labels) is often expensive, recovery the entire graph signal from a (small) subset of nodes (sampling set) is a crucial task. by, The graph signal recovery problem is possible by exploiting the tendency of natural graph signals to conform to the underlying network structure. More precisely, the clustering hypothesis, which underlies most (semi-) supervised machine learning methods $[6,7]$, requires the graph signal to be nearly constant over well connected subset of nodes (clusters).

In this paper, we further develop the program initiated in $[8,9]$ revolving around conditions which guarantee con- vex optimization methods, such as nLasso [10] or sparse label propagation [11], to accurately recover clustered graph signals from a small number of signal samples. In particular, we apply the network compatibility condition (NCC) introduced in [8] to analyze the nLasso squared error loss. We verify that the NCC ensures accurate recovery of a clustered (piece-wise constant) graph signal from only few signal values (initial labels) using nLasso.

This paper is organized as follows: In Section 2, we formalize the problem setup. In Section 3, we present our main result, i.e., that the NCC guarantees accuracy of nLasso using squared error loss if the true underlying graph signal is clustered.The results of some illustrative numerical experiments are discussed in Section 4, which also contains the ADMM update rules nLasso with squared error loss.

\section{PROBLEM FORMULATION}

In what follows we consider a dataset which is represented by its data graph $\mathcal{G}=(\mathcal{V}, \mathcal{E}, \mathbf{W})$. The nodes $\mathcal{V}=\{1, \ldots, N\}$ represent individual data points. The undirected edges $\mathcal{E}$ encode domain-specific notions of similarity between data points. The non-negative entries $W_{i, j}$ of the weight matrix $\mathbf{W} \in \mathbb{R}_{+}^{N \times N}$ quantify the level of similarity between connected nodes. In particular, the weight $W_{i, j}$ is non-zero only if nodes $i, j \in \mathcal{V}$ are connected by an edge $\{i, j\} \in \mathcal{E}$.

In addition to the graph structure $\mathcal{G}$, datasets typically convey additional information, such as labels associated with individual data points $i \in \mathcal{V}$. In what follows, we represent any such label information using a graph signal $x[\cdot]: \mathcal{V} \rightarrow \mathbb{R}$, which assigns a real value $x[i] \in \mathbb{R}$ to node the $i \in \mathcal{V}$.

Our approach is based on the assumption that the graph signals $x[\cdot]$ encountered in applications are typically clustered, i.e., have small total variation (TV) [12]

$$
\|x[\cdot]\|_{\mathcal{E}}:=\sum_{\{i, j\} \in \mathcal{E}} W_{i, j}|x[j]-x[i]| .
$$

We will use the following simple model for clustered (piecewised constant) graph signals [12]:

$$
x[i]=\sum_{\mathcal{C} \in \mathcal{F}} a_{\mathcal{C}} \mathcal{I}_{\mathcal{C}}[i],
$$


with $a_{\mathcal{C}} \in \mathbb{R}$ and the indicator signal $\mathcal{I}_{\mathcal{C}}[i] \in\{0,1\}$ for subset $\mathcal{C} \in \mathcal{V}$, i.e., $\mathcal{I}_{\mathcal{C}}[i]=1$ if and only if $i \in \mathcal{C}$. The model (2) involves a partition $\mathcal{F}=\left\{\mathcal{C}_{1}, \ldots, \mathcal{C}_{|\mathcal{F}|}\right\}$ of $\mathcal{V}$ into disjoint subsets $\mathcal{C}_{l}$.

In principle our analysis applies to any partition $\mathcal{F}=$ $\left\{\mathcal{C}_{1}, \ldots, \mathcal{C}_{|\mathcal{F}|}\right\}$ used to define the model (2). However, our results are most useful if the partition conforms with the "intrinsic (cluster) structure" of the data graph $\mathcal{G}$. In particular, we consider a partition $\mathcal{F}$ such that the total weight of the cluster boundaries

$$
\partial \mathcal{F}=\left\{\{i, j\} \in \mathcal{E}: i \in \mathcal{C}, j \in \mathcal{C}^{\prime}(\neq \mathcal{C})\right\}
$$

is small compared to the total weight of intra-cluster edges $\overline{\partial \mathcal{F}}=\mathcal{E} \backslash \partial \mathcal{F}$, i.e., $\sum_{\{i, j\} \in \partial \mathcal{F}} W_{i, j} \ll \sum_{\{i, j\} \in \overline{\partial \mathcal{F}}} W_{i, j}$. As can verified easily, for such a partition, any signal of the form (2) has a small TV $\|x[\cdot]\|_{\mathcal{E}}$.

We consider the case of having access to the graph signal values $x[i]$ only for (small) subset of nodes, i.e., the sampling set $\mathcal{M}:=\left\{i_{1}, \ldots, i_{M}\right\} \subseteq \mathcal{V}$ (typically $|\mathcal{M}| \ll|\mathcal{V}|$ ). In particular, we observe

$$
y[i]=x[i]+\varepsilon[i] \text { for a sampled node } i \in \mathcal{M} .
$$

The error or noise component $\varepsilon[i]$ in (3) subsumes any data curation or labelling errors. We do not assume any probabilistic model for the noise $\varepsilon[i]$. However, for our analysis in what follows we have to require the noise is not excessively large. In particular, we require an upper bound

$$
\sum_{i \in \mathcal{M}} \tilde{x}[i] \varepsilon[i] \leq \nu\|\tilde{x}[\cdot]\|_{\mathcal{E}}
$$

to hold for any non-constant graph signal $\tilde{x}[\cdot] \in \mathbb{R}^{N}$, i.e., for which $\|\tilde{x}\|_{\mathcal{E}} \neq 0$, and some positive constant $\nu>0$.

The recovery of the entire graph signal $x[\cdot]$ from the noisy measurements $\{y[i]\}_{i \in \mathcal{M}}$ rests on the assumption that the true underlying graph signal has small TV $\|x[\cdot]\|_{\mathcal{E}}$. Moreover, the recovered graph signal $\hat{x}[\cdot]$ should agree well with the observed samples, i.e., it should incur a small empirical error

$$
\widehat{E}(\hat{x}[\cdot]):=\sum_{i \in \mathcal{M}}(\hat{x}[i]-y[i])^{2} .
$$

Our approach to recovering the underlying graph signal is based on balancing a small TV $\|\hat{x}[\cdot]\|_{\mathcal{E}}$ with a small empirical error $\widehat{E}(\hat{x}[\cdot])$, i.e.,

$$
\hat{x}[\cdot] \in \underset{\tilde{x}[\cdot] \in \mathbb{R}^{N}}{\arg \min } \widehat{E}(\tilde{x}[\cdot])+\lambda\|\tilde{x}[\cdot]\|_{\mathcal{E}}
$$

The recovery problem (6) is a convex optimization problem [13]. Indeed, the objective function of (6) is the sum of a convex quadratic function (5) and a semi-norm $\lambda\|\tilde{x}[\cdot]\|_{\mathcal{E}}$ (which is convex) [13]. The regularization parameter $\lambda$ in the recovery problem (6) allows to trade-off a small empirical error against a small TV of the recovered graph signal. Our analysis of the accuracy achieved by the recovery method (6) will focus on a particular choice for $\lambda$ (see Theorem 2 below).

\subsection{ADMM updates for nLasso}

By introducing, for each edge $\{i, j\} \in \mathcal{E}$, the auxiliary variables $z_{i j}, z_{j i}$ (which act as local copies of $\tilde{x}[i], \tilde{x}[j]$ ), the recovery problem (6) can be rewritten as (cf. (5), (1))

$$
\begin{aligned}
& \min _{\substack{\{\tilde{x}[i]\}_{i \in \mathcal{V}} \\
\left\{z_{i j}, z_{j i}\right\}_{\{i, j\} \in \mathcal{E}}}} \sum_{i \in \mathcal{M}}(\tilde{x}[i]-y[i])^{2}+\lambda \sum_{\{i, j\} \in \mathcal{E}} W_{i, j}\left|z_{i j}-z_{j i}\right| \\
& \text { s.t } \quad \tilde{x}[i]=z_{i j}, \text { for } i \in \mathcal{V}, \quad j \in \mathcal{N}(i)
\end{aligned}
$$

We solve the convex optimization (7) using the alternating direction method of multipliers (ADMM) [14]. The use of ADMM is appealing since it naturally lends to a distributed implementation in the form of message passing over the underlying data graph $\mathcal{G}$. We have summarized this message passing formulation of the ADMM iterations in Alg. 1.

In particular, ADMM is an iterative method generating a sequence $x^{(k)}[i], k=0,1, \ldots$, of signal estimates which eventually converge to a solution of (7) [14]. For a detailed derivation of the update rules we refer to [8]. In particular, for each node $i \in \mathcal{V}$, ADMM amounts to computing the update $x^{(k+1)}[i]$ by solving

$$
\begin{aligned}
x^{(k+1)}[i]= & \underset{\tilde{x}[i]}{\arg \min }\left(\mathcal{I}_{\mathcal{M}}[i](\tilde{x}[i]-y[i])^{2}\right. \\
& \left.+(\rho / 2) \sum_{j \in \mathcal{N}(i)}\left(\tilde{x}[i]-z_{i j}^{(k)}+u_{i j}^{(k)}\right)^{2}\right) .
\end{aligned}
$$

Solving (8) for a sampled node $i \notin \mathcal{M}$, yields

$$
x^{(k+1)}[i]=(1 /|\mathcal{N}(i)|) \sum_{j \in \mathcal{N}(i)}\left(z_{i j}^{(k)}-u_{i j}^{(k)}\right),
$$

whereas for a non-sampled node $i \in \mathcal{M}$,

$$
x^{(k+1)}[i]=\frac{2}{\rho|\mathcal{N}(i)|+2}\left(y[i]+\frac{\rho}{2} \sum_{j \in \mathcal{N}(i)}\left(z_{i j}^{(k)}-u_{i j}^{(k)}\right)\right) .
$$

As the notation in (6) suggests, there might be several solutions $\hat{x}$ which achieve the minimum objective value. However, our analysis applies to any of those optimal solutions so that this non-uniqueness poses no difficulty for our approach. The tuning parameter $\lambda$ in (6) trades off a small empirical error $\widehat{E}(\hat{x}[\cdot])$ (cf. (5)) against a small total variation $\|\hat{x}[\cdot]\|_{\mathcal{E}}$ (cf. (1)) of the recovered signal $\hat{x}[\cdot]$. A small value of $\lambda$ enforces the solutions of (6) to achieve a small empirical error, whereas, a large value of $\lambda$ enforces the solutions of (6) to have a small TV.

The recovery problem (6) is a convex problem and can be approached by modern convex optimization methods $[11,15$, 16]. These optimization methods take advantage of a particular structure which is presented in the nLasso problem (6). In particular, the nLasso amounts to minimizing the sum of two functions, each of which could be minimized easily when considered isolated. 


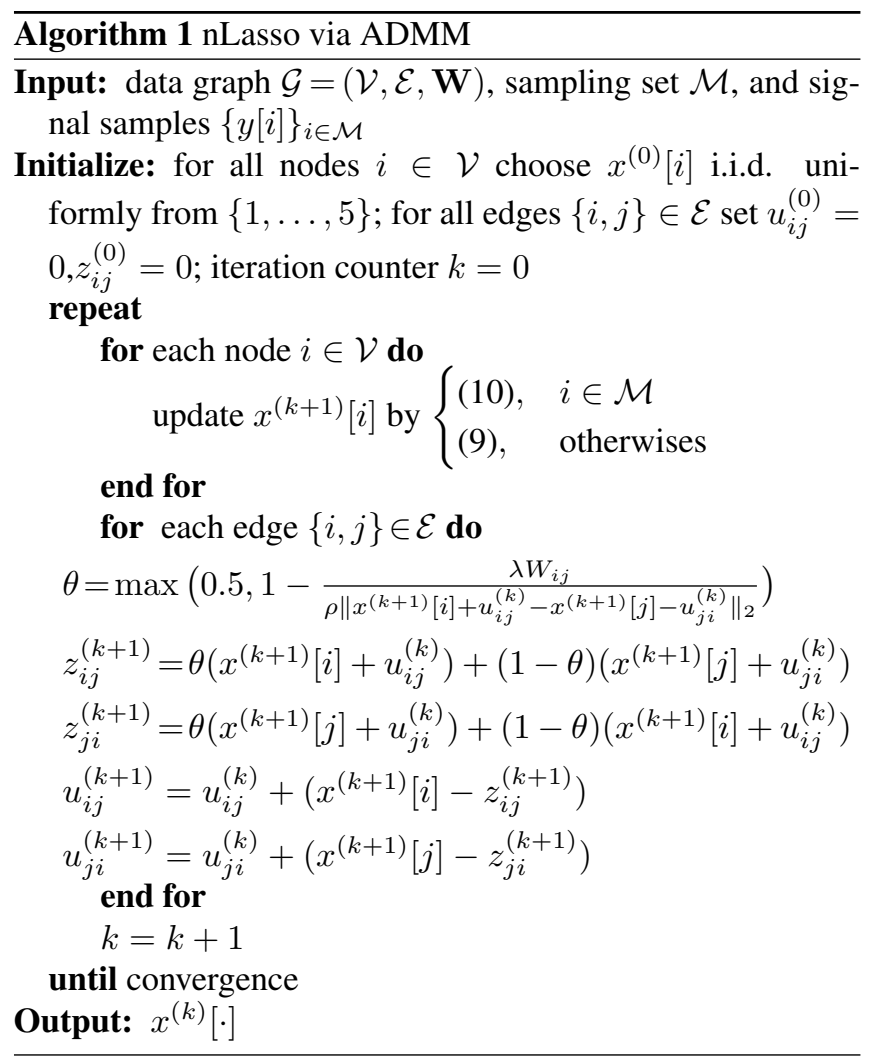

\section{WHEN IS NETWORK LASSO ACCURATE?}

Our main contribution is to show that the NCC guarantees any solutions of (6) allows to accurately recover the true underlying graph signal.

Definition 1. Consider a data graph $\mathcal{G}=(\mathcal{V}, \mathcal{E}, \mathbf{W})$ with a particular partition $\mathcal{F}$ of its nodes $\mathcal{V}$. A sampling set $\mathcal{M} \subseteq \mathcal{V}$ is said to satisfy NCC with constants $K, L>0$, if

$$
L\|z[\cdot]\|_{\partial \mathcal{F}} \leq K \sqrt{\sum_{i \in \mathcal{M}}(z[i])^{2}}+\|z[\cdot]\| \frac{}{\partial \mathcal{F}}
$$

for any graph signal $z[\cdot] \in \mathbb{R}^{N}$.

It turns out that, if the sampling set satisfies the NCC, any solution of (6) provides an accurate estimate of the true underlying graph signal (2).

Theorem 2. Consider a data set with data graph $\mathcal{G}$ and $a$ graph signal $x[\cdot]$ of the form (2) with underlying partition $\mathcal{F}$. We observe noisy signal value on the sampling set $\mathcal{M}$. The sampling set $\mathcal{M}$ is chosen such that it satisfies NCC with parameters $L>4$ and $K>0$. The noise is sufficiently small such that (4) holds with some noise level $\nu<1 /(4 K)$. Then any solution $\hat{x}[\cdot]$ of $n$ Lasso (6) with $\lambda:=1 / K$, satisfies

$$
\|\hat{x}[\cdot]-x[\cdot]\|_{\mathcal{E}} \leq 12 K .
$$

Proof of Theorem 2. Consider an arbitrary solution $\hat{x}[\cdot]$ of (6) and denote the difference between $\hat{x}[\cdot]$ and the true underlying clustered signal $x[\cdot]$ as $\tilde{x}[\cdot]:=\hat{x}[\cdot]-x[\cdot]$, which we assume to be non-constant, i.e., $\|\tilde{x}[\cdot]\|_{\mathcal{E}} \neq 0$. By (6),

$$
\sum_{i \in \mathcal{M}}(\hat{x}[i]-y[i])^{2}+\lambda\|\hat{x}[\cdot]\|_{\mathcal{E}} \leq \sum_{i \in \mathcal{M}} \varepsilon[i]^{2}+\lambda\|x[\cdot]\|_{\mathcal{E}} .
$$

Using the decomposition property for the semi-norm $\|\cdot\|_{\mathcal{E}}$,

$$
\begin{aligned}
& \sum_{i \in \mathcal{M}}(\hat{x}[i]-y[i])^{2}+\lambda\|\hat{x}[\cdot]\| \frac{}{\partial \mathcal{F}} \\
& \leq \sum_{i \in \mathcal{M}} \varepsilon[i]^{2}+\lambda\left(\|x[\cdot]\|_{\partial \mathcal{F}}-\|\hat{x}[\cdot]\|_{\partial \mathcal{F}}\right)
\end{aligned}
$$

Since $x[\cdot]$ is assumed clustered (cf. (2)),

$$
\|x[\cdot]\| \frac{}{\partial \mathcal{F}}=0 \text {, and }\|\tilde{x}[\cdot]\|_{\overline{\partial \mathcal{F}}}=\|\hat{x}[\cdot]\|_{\overline{\partial \mathcal{F}}} .
$$

Thus, inserting (15) into (14), by the triangle inequality

$$
\sum_{i \in \mathcal{M}}(\hat{x}[i]-y[i])^{2}+\lambda\|\tilde{x}[\cdot]\| \frac{}{\partial \mathcal{F}} \leq \sum_{i \in \mathcal{M}} \varepsilon[i]^{2}+\lambda\|\tilde{x}[\cdot]\|_{\partial \mathcal{F}}
$$

Combining (16) with

$$
\begin{aligned}
\sum_{i \in \mathcal{M}}(\hat{x}[i] & -y[i])^{2} \stackrel{(3)}{=} \sum_{i \in \mathcal{M}}(\tilde{x}[i]-\varepsilon[i])^{2} \\
& =\sum_{i \in \mathcal{M}} \tilde{x}[i]^{2}+\sum_{i \in \mathcal{M}} \varepsilon[i]^{2}-2 \sum_{i \in \mathcal{M}} \tilde{x}[i] \varepsilon[i] \\
& \stackrel{(4)}{\geq} \sum_{i \in \mathcal{M}} \tilde{x}[i]^{2}+\sum_{i \in \mathcal{M}} \varepsilon[i]^{2}-2 \nu\|\tilde{x}[\cdot]\| \|_{\mathcal{E}}
\end{aligned}
$$

yields

$$
\sum_{i \in \mathcal{M}} \tilde{x}[i]^{2}+\lambda\|\tilde{x}[\cdot]\|\left\|_{\overline{\partial \mathcal{F}}} \leq 2 \nu\right\| \tilde{x}[\cdot]\left\|_{\mathcal{E}}+\lambda\right\| \tilde{x}[\cdot] \|_{\partial \mathcal{F}} .
$$

Thus, since $\lambda=1 / K$ and $\nu<1 /(4 K)$, inequality (18) implies that the difference signal $\tilde{x}[\cdot]$ is approximately clustered according to $\mathcal{F}$, i.e.,

$$
\|\tilde{x}[\cdot]\|_{\partial \mathcal{F}} \leq 3\|\tilde{x}[\cdot]\|_{\partial \mathcal{F}}
$$

Since $\mathcal{M}$ satisfies NNC with $L>4$, (11) and (19) yield

$$
(1 / K)^{2}\|\tilde{x}[\cdot]\|_{\partial \mathcal{F}}^{2} \leq \sum_{i \in \mathcal{M}} \tilde{x}[i]^{2}
$$

Combining (20) with (18) yields (12).

\section{NUMERICAL RESULTS}

We now present numerical experiments to illustrate our theoretical results in Section 3. These experiments revolve around a synthetic dataset which is generated using the BarabásiAlbert (BA) model [17]. The resulting data graph is partitioned into 5 disjoint clusters $\mathcal{F}=\left\{\mathcal{C}_{1}, \ldots, \mathcal{C}_{5}\right\}$, each cluster consisting of 100 nodes.

In particular, starting from an initially connected network of 20 nodes, new nodes are added to the network one at a time and are connected to three existing nodes until obtaining a cluster of 100 nodes. The process is repeated 5 times in order to obtain 5 different clusters. These clusters are connected by randomly adding a total of 250 inter-cluster edges (see Fig. 
1). The edge weights of those inter-cluster edges $\{i, j\} \in \partial \mathcal{F}$ are set uniformly as $W_{i j}=2$. In contrast, an edge connecting two nodes $i, j \in \mathcal{C}_{k}$ from the same cluster $\mathcal{C}_{k}$ is assigned the weight $W_{i j}=10 k$.

We then generate a clustered graph signal according to (2) using the signal coefficients $a_{\mathcal{C}_{k}}=k$ (see Fig. 1). A noisy version of the signal values are observed at the nodes in the sampling set $\mathcal{M}$ which is constructed by randomly selecting 50 nodes (which are shown in Fig. 2). In particular, the observed signal values are generated according to observation model (3) with the noise values $\varepsilon[i]$ being i.i.d. zeromean Gaussian random variables with variance $\sigma^{2}=0.2$, i.e., $\varepsilon[i] \sim \mathcal{N}(0,0.2)$. In order to recover the entire graph signal from the noisy signal samples $\{y[i]\}_{i \in \mathcal{M}}$, we run Alg. 1 to solve the recovery problem (6).

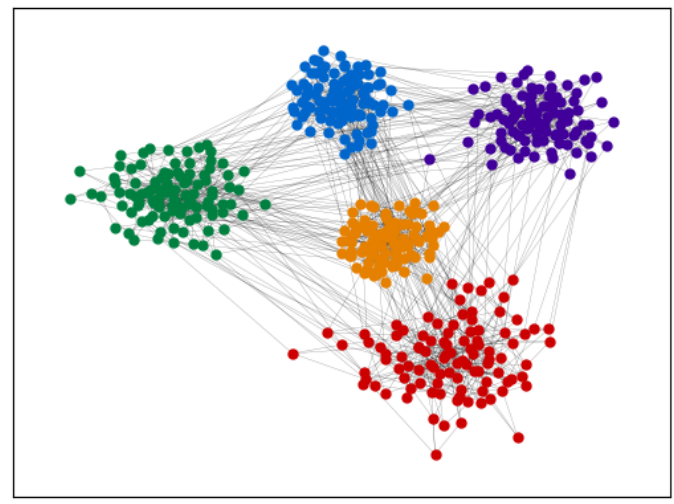

Fig. 1. A data graph obtained from the BA model which is partitioned into 5 clusters. The nodes belonging to the same cluster are coloured likely.

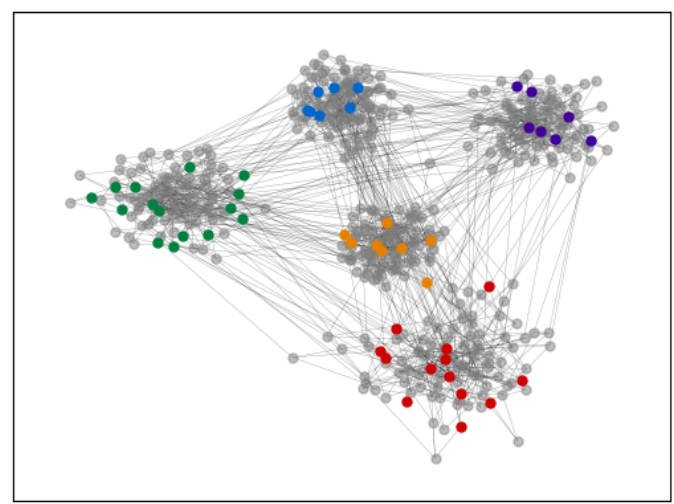

Fig. 2. The coloured nodes form the sampling set $\mathcal{M}$.

In particular, we run Alg. 1 with a manually tuned parameter $\lambda=10^{-3}$ and $\rho=5 \cdot 10^{-2}$, for a fixed number of 200 iterations. The result is post-processed by rounding the recovered signal to the nearest integer and is plotted in Fig. 3. According to Fig. 3, Alg. 1 is able to perfectly recover the

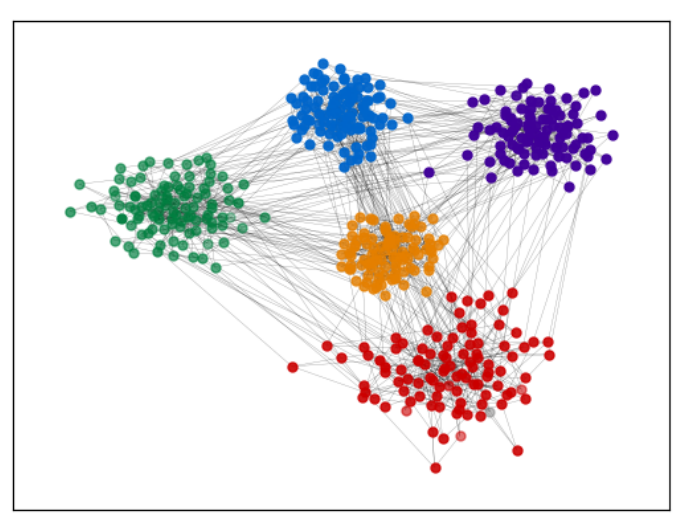

Fig. 3. The recovered graph signal obtained from Alg. 1 (each colour corresponds to one particular signal value).

clustered-structure of the underlying graph signal. In Fig.4, we plot the objective value as a function of iteration number which indicates that the Alg. 1 converges in less than 50 iterations. We also computed, using 1000 i.i.d. simulation runs, the normalized mean square error yielding a value of $9.99 \cdot 10^{-3}$ (standard deviation $6.4 \cdot 10^{-3}$ ).

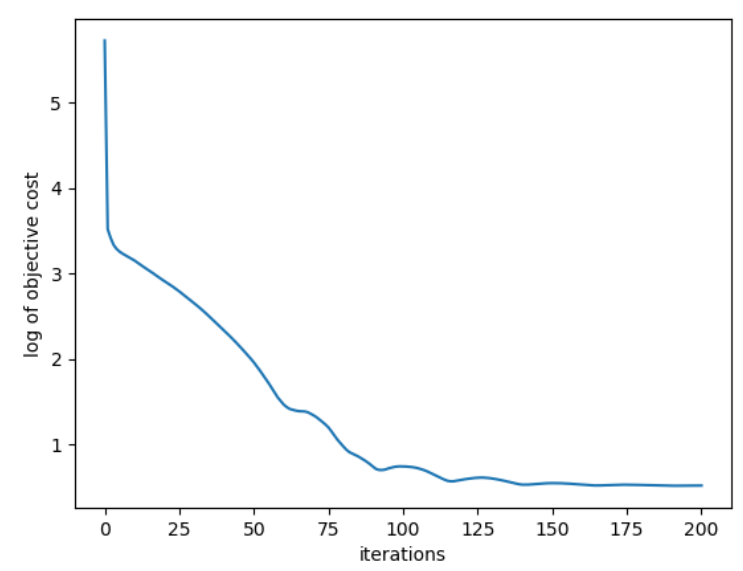

Fig. 4. The objective value of the ADMM iterates in Algorithm 1.

\section{REFERENCES}

[1] A. Sandryhaila and J. M. F. Moura, "Classification via regularization on graphs," in 2013 IEEE Global Conference on Signal and Information Processing, Dec 2013, pp. 495-498.

[2] A. Jung, G. Hannak, and N. Görtz, "Graphical LASSO Based Model Selection for Time Series," IEEE Sig. Proc. Letters, vol. 22, no. 10, pp. 1781-1785, Oct. 2015. 
[3] A. Jung, "Learning the conditional independence structure of stationary time series: A multitask learning approach," IEEE Trans. Signal Processing, vol. 63, no. 21, Nov. 2015.

[4] N. Q. Tran and A. Jung, "Learning conditional independence structure for high-dimensional uncorrelated vector processes," New Orleans (LA), 2017, pp. 59205924.

[5] N. Q. Tran and A. Jung, "On the sample complexity of graphical model selection for non-stationary processes," in Proc. IEEE ICASSP-2018, Calgary (CN), Apr. 2018.

[6] C. M. Bishop, Pattern Recognition and Machine Learning, Springer, 2006.

[7] O. Chapelle, B. Schölkopf, and A. Zien, Eds., SemiSupervised Learning, The MIT Press, Cambridge, Massachusetts, 2006.

[8] A. Jung, N. Q. Tran, and A. Mara, "When is network lasso accurate?," Front. Appl. Math. Stat., Jan. 2018.

[9] A. Jung, A. Heimowitz, and Y. C. Eldar, "The network nullspace property for compressed sensing over networks," in Proc. Int. Conf. Sampling Th. and Applications (SampTA).

[10] D. Hallac, J. Leskovec, and S. Boyd, "Network lasso: Clustering and optimization in large graphs," in Proc. SIGKDD, 2015, pp. 387-396.

[11] A. Jung and M. Hulsebos, "The network nullspace property for compressed sensing of big data over networks," Front. Appl. Math. Stat., Apr. 2018.

[12] S. Chen, Y. Yang, J. M. F. Moura, and J. Kovačević, "Signal localization, decomposition and dictionary learning on graphs," arxiv:1607.01100, 2017.

[13] S. Boyd and L. Vandenberghe, Convex Optimization, Cambridge Univ. Press, Cambridge, UK, 2004.

[14] S. Boyd, N. Parikh, E. Chu, B. Peleato, and J. Eckstein, Distributed Optimization and Statistical Learning via the Alternating Direction Method of Multipliers, vol. 3 of Foundations and Trends in Machine Learning, Now Publishers, Hanover, MA, 2010.

[15] A. Jung, P. Berger, G. Hannak, and G. Matz, "Scalable graph signal recovery for big data over networks," in 2016 IEEE 17th International Workshop on Signal Processing Advances in Wireless Communications (SPAWC), July 2016, pp. 1-6.

[16] G. Hannak, P. Berger, G. Matz, and A. Jung, "Efficient graph signal recovery over big networks," in Proc. Asilomar Conf. Signals, Sstems, Computers, Nov 2016, pp. 1-6.
[17] B. Bollobas, W. Fulton, A. Katok, F. Kirwan, and P. Sarnak, Random graphs, vol. 73, Cambridge studies in advanced mathematics., 2001. 